OPEN ACCESS

Edited by:

Alexey Goltsov,

Abertay University, UK

Reviewed by:

Karen Yvonne Stokes,

Louisiana State University Health

Sciences Center, USA

Wang Min,

Yale University, USA

*Correspondence:

Yan-Yan Li

Iyynjmu123@126.com

Specialty section: This article was submitted to

Vascular Physiology,

a section of the journal

Frontiers in Physiology

Received: 30 December 2015 Accepted: 23 May 2016

Published: 09 June 2016

Citation:

Li Y Y Y, Yang $X-X$, Zhou $Y-H$, Gong $G$, Geng H-Y, Kim HJ, Zhou C-W, Qian Y,

Wang X-M and Wu J (2016) Matrix

metalloproteinase-9 Gene-1562C>T

Gene Polymorphism and Coronary

Artery Disease in the Chinese Han

Population: A Meta-Analysis of 5468

Subjects. Front. Physiol. 7:212.

doi: 10.3389/fphys.2016.00212

\title{
Matrix metalloproteinase-9 Gene-1562C > T Gene Polymorphism and Coronary Artery Disease in the Chinese Han Population: A Meta-Analysis of 5468 Subjects
}

Yan-Yan $\mathrm{Li}^{1 *}$, Xin-Xing Yang ${ }^{1}$, Yan-Hong Zhou ${ }^{1}$, Ge Gong ${ }^{1}$, Hong-Yu Geng ${ }^{1}$, Hyun $\mathrm{J} \mathrm{Kim}^{2}$,
Chuan-Wei Zhou ${ }^{1}$, Yun Qian ${ }^{1}$, Xiang-Ming Wang ${ }^{1}$ and Jun Wu ${ }^{1}$

${ }^{1}$ Department of Geriatrics, First Affiliated Hospital of Nanjing Medical University, Nanjing, China, ${ }^{2}$ Department of Radiation Oncology, University of Pennsylvania, Philadelphia, PA, USA

Background: Multiple studies indicate that the matrix metalloproteinase-9 (MMP-9)-1562C>T gene polymorphism may be associated with an increased risk of coronary artery disease (CAD) in the Chinese Han population. However, a clear consensus has yet to be established.

Objective and methods: A meta-analysis of 5468 subjects from 10 separate studies was performed to explore the possible relationship between the MMP-9-1562C>T gene polymorphism and CAD within the Chinese Han population. Pooled odds ratio (ORs) for the association and the corresponding 95\% confidence intervals (Cls) were evaluated by a random or fixed-effect model.

Results: Our analysis confirms the association between the MMP-9-1562C >T gene polymorphism and an increased risk of CAD within the Chinese Han population under allelic (OR: 1.60, 95\% Cl: 1.25-2.04, $P=0.0002$ ), recessive (OR: 3.05, 95\% Cl: 1.67-5.56, $P=0.0003$ ), dominant (OR: 2.23, 95\% Cl: 1.49-3.35, $P=0.0001$ ), homozygous (OR: $3.41,95 \% \mathrm{Cl}: 1.87-6.23, P<0.0001)$, heterozygous (OR: $2.03,95 \%$ Cl: 1.40-2.93, $P=0.0002$ ), and additive genetic models (OR: 1.78, 95\% Cl: 1.33-2.39, $P<0.0001)$.

Conclusions: In the Chinese Han population, the MMP-9-1562C $>T$ gene polymorphism is correlated with an increased risk of CAD. Therefore, Han Chinese carriers of the $-1562 T$ allele may be at an increased risk of CAD.

Keywords: matrix metalloproteinase-9, $-1562 \mathrm{C}>\mathrm{T}$, polymorphism, coronary artery disease, Chinese

\section{INTRODUCTION}

Coronary artery disease (CAD) is a chronic condition with both hereditary and environmental factors. The increasing prevalence of unhealthy lifestyles in China (i.e., extended periods of sitting, a more sedentary lifestyle, a high-fat diet), the general aging of the Chinese population, and the increasing rates of hypertension and diabetes have damaged the quality of life of many Chinese 
people and sharply increased CAD-associated morbidity and mortality (Li, 2007). However, recent advancements in molecular biology have allowed researchers to better elucidate the pathogenic mechanism of CAD.

Matrix metalloproteinases (MMPs), a large family of zincdependent proteolytic enzymes that have special functions in extracellular matrix (ECM) degradation have been a particular rich area of research. MMPs play a role in multiple pathophysiological processes such as ECM degradation, inflammation response, tumor metastasis, and atherosclerosis (Castro and Tanus-Santos, 2013). Enhanced MMP expression and activity also play a pivotal role in early arteriosclerosis, plaque rupture, myocardial infarction, and heart failure (Tanner et al., 2011). MMP-9 (also known as gelatinase B) is one the most extensively researched MMPs and exhibits enhanced expression in atherosclerosis injury sites. It has been also found that there were many gene sequence variations in $M M P-9$ gene, of which the locus rs3918242 $(-1562 \mathrm{C}>\mathrm{T})$ in the promoter region was the most reported gene locus.

MMP-9 gene, located in 20q11.2-13.1, spans $7.7 \mathrm{~kb}$ and contains 13 exons. MMP-9-1562C $>\mathrm{T}$ mutation occurs in a substitution of cytosine (C) for thymine (T). This mutation may increase $M M P-9$ gene expression and risk of CAD development by reducing the binding affinity with transcription inhibition proteins.

Although many studies on the association between MMP-9$1562 \mathrm{C}>\mathrm{T}$ gene polymorphism and CAD have been performed in China, the composite of these studies fail to provide a consensus. In 2005, Tang et al found that MMP-9-1562C > T gene polymorphism was significantly associated with CAD in a Zhejiang population and $\mathrm{T}$ allele increased the CAD risk (Tang et al., 2005). In 2007, Chen et al also reported a similar result in another Hunan population (Chen et al., 2007). In contrast, Wu et al failed to find an association between MMP-9-1562C $>\mathrm{T}$ gene polymorphism and CAD in a Beijing population in 2009 (Wu et al., 2009) and Zhi et al observed no significant effects for MMP-9-1562C > T gene polymorphism on CAD risk in a Jiangsu population (Zhi et al., 2010).

We performed the current meta-analysis with the hopes to provide a valuable conclusion on the association between $M M P$ 9-1562C > T gene polymorphism and CAD in the Chinese Han population.

\section{MATERIALS AND METHODS}

\section{Publication Search and Inclusion Criteria}

The Web of Science, PubMed, Embase, the China Biological Medicine Database, and the China National Knowledge Infrastructure electronic databases were searched using the terms "matrix metalloproteinase-9," "coronary artery disease," or "coronary heart disease," and "polymorphism" in our initial search. Retrieved studies were published between 2005 and 2010 with the last study updated on March 26, 2016.

To meet our inclusion criteria, studies had to (a) evaluate the association between $M M P-9-1562 \mathrm{C}>\mathrm{T}$ gene polymorphism and CAD in the Chinese Han population. (b) diagnose CAD according to the clinical symptoms combined with examination results (i.e., coronary arteriography, electrocardiogram, treadmill exercise test, echocardiography, myocardial perfusion imaging by Emission Computed Tomography, etc.) with a minimal stenosis rate of the major coronary artery diameter of more than $50 \%$ (c) be a case-control or cohort study published in an official journals or as a postgraduate dissertation.

\section{Data Extraction}

Data was extracted according to a standardized protocol. Two investigators searched for duplicates while a third served as an arbiter to resolve possible disagreements. Duplicate papers, those that violated the inclusion criteria, or those that provided deficient data were removed. Identical data sets used in different studies by the same authors were used once. Abstracted data consisted of the following items: the first author's name, publication year, region, number of genotypes, genotyping method, study design, age, gender, and total number of cases and controls.

\section{Statistical Analysis}

The odds ratio (OR) and its corresponding to 95\% confidence interval (CI) were used to measure the association between MMP-9-1562C > T gene polymorphism and CAD. The Chisquare-based Q-test was adopted to measure the effects betweenstudies heterogeneity $(P<0.05$ level) (Cochran, 1968).Variation due to heterogeneity was assessed by the inconsistency index $I^{2}$. If heterogeneity were present in the study, the randomeffects model would be used to assess the combined OR (the DerSimonian and Laird method) (DerSimonian and Laird, 1986). Otherwise, the fixed-effects model would be used (the MantelHaenszel method) (Mantel and Haenszel, 1959). The pooled OR was determined by $Z$-test and significance was set at $P<0.05$.

The Fisher's exact test was used to evaluate the HardyWeinberg equilibrium (HWE) $(P<0.05)$. Potential publication bias was assessed by Egger's linear regression test on the natural $\log$ scale of the OR to detect funnel plot asymmetry $(P<0.05$ level) (Egger et al., 1997). The statistical analysis was performed by STATA 11.0 software (StataCorp, College Station, TX).

\section{RESULTS}

\section{Studies and Populations}

Ten of the nineteen retrieved papers fit the inclusion criteria. Of the nine excluded studies, one was a duplicate, four were reviews, and another four were irrelevant to our interests. No study was excluded for deviation from HWE. The ten studies compiled the data from 3168CAD patients and 2300 controls (Table 1, Presentation 1 in Supplementary Material; Tang et al., 2005; Meng et al., 2006; Chen et al., 2007; Wang et al., 2007; Wu et al., 2009; Gao and Wang, 2010; Ma et al., 2010; Yong and Shi, 2010; Zhang et al., 2010; Zhi et al., 2010) and represented seven provinces (Shanxi, Xinjiang, Jiangsu, Zhejiang, Hunan, Tianjin, and Beijing). All subjects were of Han ethnicity. However, there were substantial differences in the size of the total patient population between these studies. The Wu $\mathrm{N}$ study alone contributed $1356 \mathrm{CAD}$ patients and 689 controls for this 
TABLE 1 | Characteristics of the investigated studies of the association between the matrix metalloproteinase-9 gene $-1562 \mathrm{C}>\mathrm{T}$ polymorphism and coronary artery disease in the Chinese population.

\begin{tabular}{|c|c|c|c|c|c|c|c|c|c|c|c|c|c|}
\hline \multirow[t]{2}{*}{ Author } & \multirow[t]{2}{*}{ Year } & \multirow[t]{2}{*}{ Region } & \multicolumn{3}{|c|}{ CAD } & \multicolumn{3}{|c|}{ Control } & \multicolumn{2}{|c|}{ Age (years old) } & \multicolumn{2}{|c|}{ Gender (female/male) } & \multirow{2}{*}{$\begin{array}{l}\text { Sample size } \\
\text { (CAD/control) }\end{array}$} \\
\hline & & & CC & CT & TT & CC & CT & TT & CAD & Control & CAD & Control & \\
\hline Tang et al. & 2005 & Zhejiang & 73 & 27 & 1 & 91 & 13 & 1 & $64.1 \pm 10.7$ & $62.3 \pm 11.2$ & $73 / 28$ & $86 / 19$ & $101 / 105$ \\
\hline Meng et al. & 2006 & Tianjin & 91 & 26 & 0 & 80 & 18 & 1 & $56.4 \pm 7.7$ & $54.3 \pm 8.1$ & $40 / 77$ & $32 / 67$ & $117 / 99$ \\
\hline Chen et al. & 2007 & Hunan & 63 & 25 & 2 & 58 & 11 & 1 & $59.2 \pm 10.4$ & $57.7 \pm 10.2$ & $57 / 33$ & $44 / 26$ & $90 / 70$ \\
\hline Wang et al. & 2007 & Shanxi & 46 & 17 & 1 & 66 & 18 & 0 & $63.08 \pm 13.3$ & $62.55 \pm 9.2$ & $43 / 21$ & $52 / 32$ & $64 / 84$ \\
\hline Wu et al. & 2009 & Beijing & 1078 & 263 & 15 & 545 & 143 & 1 & $58.39 \pm 10.84^{*}$ & $60.42 \pm 9.07$ & $1162 / 194$ & $589 / 100$ & $1356 / 689$ \\
\hline Gao and Wang & 2010 & Jiangsu & 49 & 38 & 9 & 59 & 18 & 1 & $64.45 \pm 9.88$ & $62.74 \pm 9.17$ & $59 / 37$ & 45/33 & $96 / 78$ \\
\hline Ma et al. & 2010 & Xinjiang & 266 & 84 & 12 & 348 & 67 & 4 & $56.7 \pm 9.4$ & $55.2 \pm 10.1$ & $249 / 113$ & 292/127 & $362 / 419$ \\
\hline Yong and Shi & 2010 & Zhejiang & 97 & 30 & 1 & 92 & 14 & 0 & $64.0 \pm 10.47$ & $61.0 \pm 11.22$ & $74 / 54$ & $65 / 41$ & 128/106 \\
\hline Zhang et al. & 2010 & Shanxi & 67 & 22 & 3 & 83 & 12 & 0 & $54.50 \pm 6.4$ & $52.30 \pm 6.9$ & $62 / 30$ & $59 / 36$ & $92 / 95$ \\
\hline Zhi et al. & 2010 & Jiangsu & 585 & 174 & 3 & 442 & 110 & 3 & $67.46 \pm 9.61$ & $69.90 \pm 11.48$ & $543 / 219$ & $372 / 183$ & $762 / 555$ \\
\hline
\end{tabular}

CAD, coronary artery disease.

Polymerase chain reaction-restriction fragment length polymorphism genotyping method and Case-control study design were adopted in the above studies.

Compared with control group, ${ }^{*} P<0.05$.

TABLE 2 | Summary of meta-analysis of association of matrix metalloproteinase-9 gene $-1562 \mathrm{C}>\mathrm{T}$ polymorphism and coronary artery disease in the Chinese population.

\begin{tabular}{|c|c|c|c|c|c|c|}
\hline Genetic model & Pooled OR (95\% Cl) & $P$-value & Literature number & CAD size & Control size & $P_{\text {heterogeneity }}\left(I^{2} \%\right)$ \\
\hline Allelic genetic model & $1.60(1.25-2.04)$ & $0.0002^{\star}$ & 10 & 3168 & 2300 & $0.002^{\star}(65.1 \%)$ \\
\hline Subgroup 1: CT1<30 & $1.74(1.28-2.36)$ & $0.0004^{\star}$ & 5 & 464 & 453 & $0.38(4.0 \%)$ \\
\hline Subgroup 2: CT1 $\geq 30$ & $1.52(1.10-2.11)$ & $0.01^{*}$ & 5 & 2704 & 1847 & $0.001^{\star}(78.3 \%)$ \\
\hline Recessive genetic model & $3.05(1.67-5.56)$ & $0.0003^{\star}$ & 10 & 3168 & 2300 & $0.50(0 \%)$ \\
\hline Dominant genetic model & $2.23(1.49-3.35)$ & $0.0001^{*}$ & 10 & 3168 & 2300 & $<0.00001^{\star}(83.5 \%)$ \\
\hline Subgroup 1: CC0>90 & $1.73(1.13-2.65)$ & $0.01^{*}$ & 5 & 2709 & 1874 & $<0.0001^{\star}(83.6 \%)$ \\
\hline Subgroup 2: CCO <90 & $3.40(1.41-8.21)$ & $0.006^{\star}$ & 5 & 459 & 426 & $0.0003^{\star}(80.9 \%)$ \\
\hline Homo genetic model & $3.41(1.87-6.23)$ & $<0.0001^{*}$ & 10 & 3168 & 2300 & $0.43(1.1 \%)$ \\
\hline Hetero genetic model & $2.03(1.40-2.93)$ & $0.0002^{*}$ & 10 & 3168 & 2300 & $<0.00001^{\star}(80.3 \%)$ \\
\hline Subgroup 1: $T 1<110$ & $3.19(1.95-5.21)$ & $<0.00001^{\star}$ & 5 & 443 & 432 & $0.12(45.5 \%)$ \\
\hline Subgroup 2: T1>110 & $1.41(1.00-2.01)$ & $0.05^{\star}$ & 5 & 2725 & 1868 & $0.003^{\star}(75.1 \%)$ \\
\hline Additive genetic model & 1.78 (1.33-2.39) & $<0.0001^{*}$ & 10 & 3168 & 2300 & $<0.0001^{\star}(75.9 \%)$ \\
\hline Subgroup 1: CT1<30 & 1.89 (1.34-2.68) & $0.0003^{*}$ & 5 & 464 & 453 & $0.26(23.7 \%)$ \\
\hline Subgroup 2: CT1 $\geq 30$ & $1.72(1.14-2.57)$ & $0.009^{*}$ & 5 & 2704 & 1847 & $<0.0001^{\star}(85.8 \%)$ \\
\hline
\end{tabular}

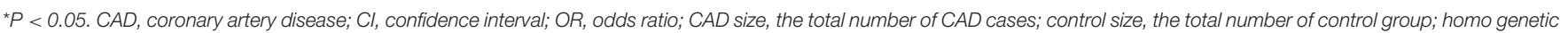

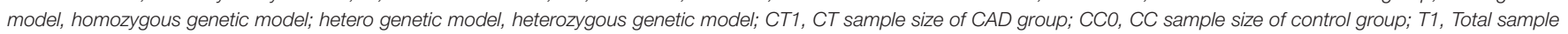
size of $C A D$ group.

meta-analysis. Differences in patient number may be factor in the lack of consensus on this topic.

\section{Combined Analyses}

There was a significant association between MMP-9-1562C > T gene polymorphism and CAD in the Chinese Han population under allelic (OR: 1.60, 95\% CI: 1.25-2.04, $P=0.0002$ ), recessive (OR: 3.05, 95\% CI: 1.67-5.56, $P=0.0003$ ), dominant (OR: 2.23, 95\% CI: $1.49-3.35, P=0.0001$ ), homozygous (OR: 3.41, 95\% CI: 1.87-6.23, $P<0.0001$ ), heterozygous (OR: 2.03, 95\% CI: $1.40-2.93, P=0.0002$ ), and additive genetic models (OR: 1.78, 95\% CI: 1.33-2.39, $P<0.0001)$. (Table 2, Figures 1-6).

There was also significant heterogeneity under the allelic $\left(P=0.002, I^{2}=65.1 \%\right)$, dominant $\left(P<0.00001, I^{2}=83.5 \%\right)$, heterozygous $\left(P<0.00001, I^{2}=80.3 \%\right)$ and additive genetic models $\left(P<0.0001, I^{2}=75.9 \%\right)$. Subsequent meta-regressions explored the source of this heterogeneity under their respective genetic models.

Under the allelic genetic model, $\mathrm{CC}$ sample size of $\mathrm{CAD}$ group (CC1, $P=0.006$ ), CT sample size of CAD group (CT1, $P=0.005)$, TT sample size of CAD group (TT1, $P=0.010$ ), CC sample size of control group (CC0, $P=0.023)$, total sample size of control group (T0, $P=0.034$ ), and weight (\%) $(P=0.030)$ were possible confounding factors that could partially explain heterogeneity between studies.

Under the additive genetic model, CC1 $(P=0.013), \mathrm{CT} 1(P=$ $0.012)$, and TT1 $(P=0.016)$ were the confounding factors. In the allelic and additive genetic models, CT1 plays a central role 


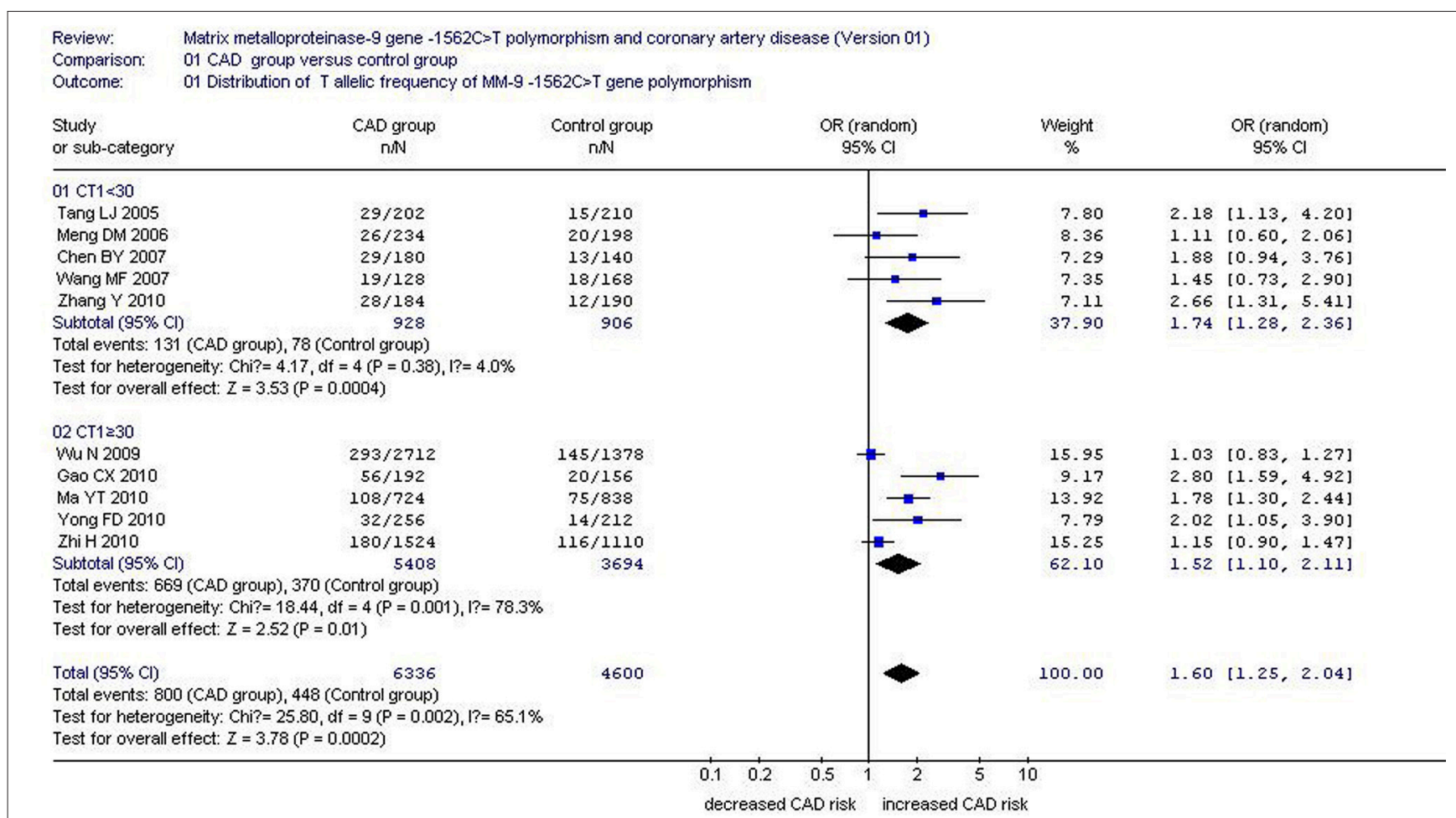

FIGURE 1 | Forest plot of coronary artery disease associated with MMP-9-1562C > T gene polymorphism under an allelic genetic model stratified by CT1 (distribution of T allelic frequency of MMP-9 gene).

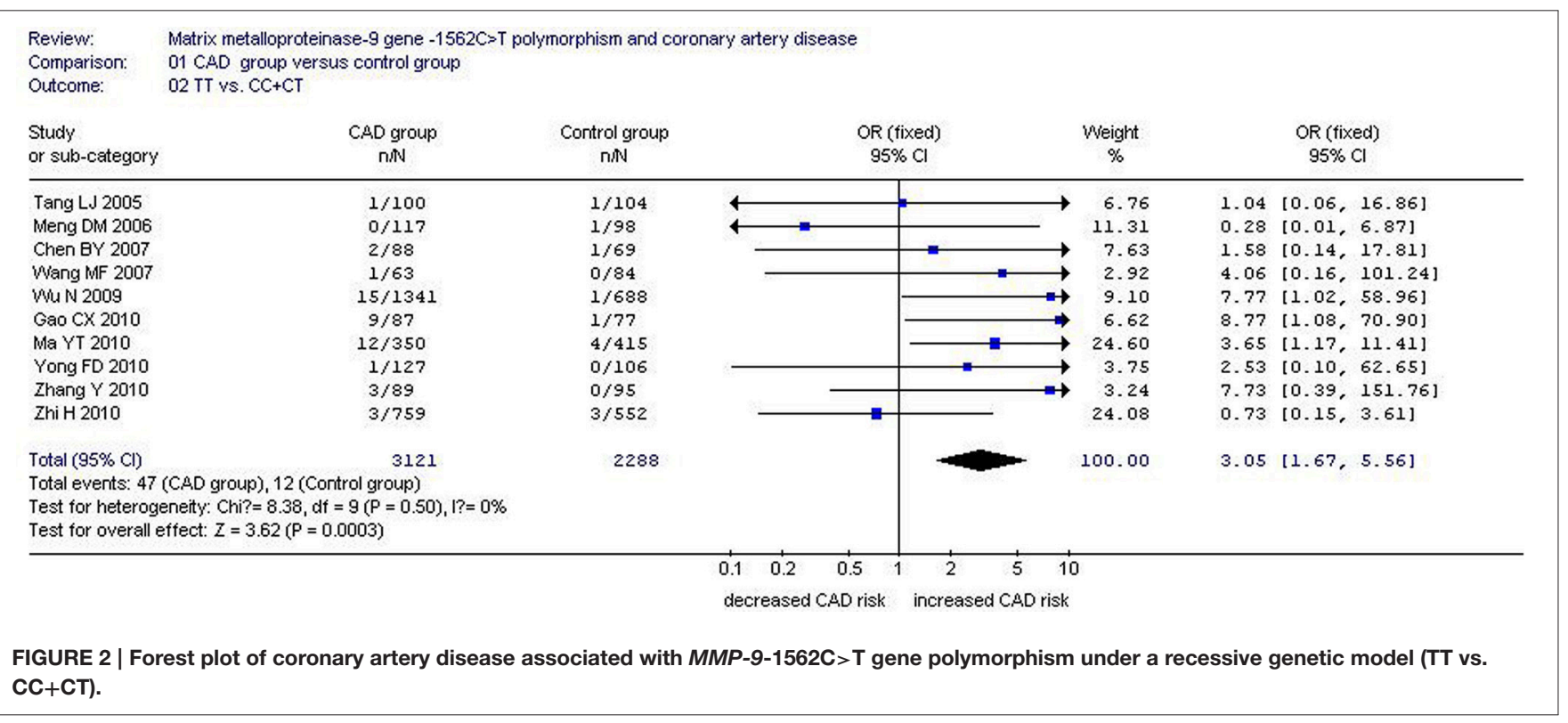

in explaining the source of heterogeneity. According to CT1, the whole population was separated into two subgroups. The studies with CT1 $<30$ were grouped into subgroup 1 and the residual studies with CT1 $\geq 60$ belonged to subgroup 2 .

Stratified by CT1, a subgroup analysis under allelic and additive genetic models found significant association between
MMP-9-1562C > T gene polymorphism and CAD in both subgroups (allelic genetic model: subgroup 1: OR: 1.74, 95\% CI: $1.28-2.36, \quad P=0.0004$; subgroup 2: OR: $1.52,95 \%$ CI: $1.10-2.11, P=0.01$ ) (additive genetic model: subgroup 1: OR: $1.89,95 \% \mathrm{CI}: 1.34-2.68, P=0.0003$; subgroup 2: OR: $1.72,95 \%$ CI: $1.14-2.57, P=0.009)$. No significant 


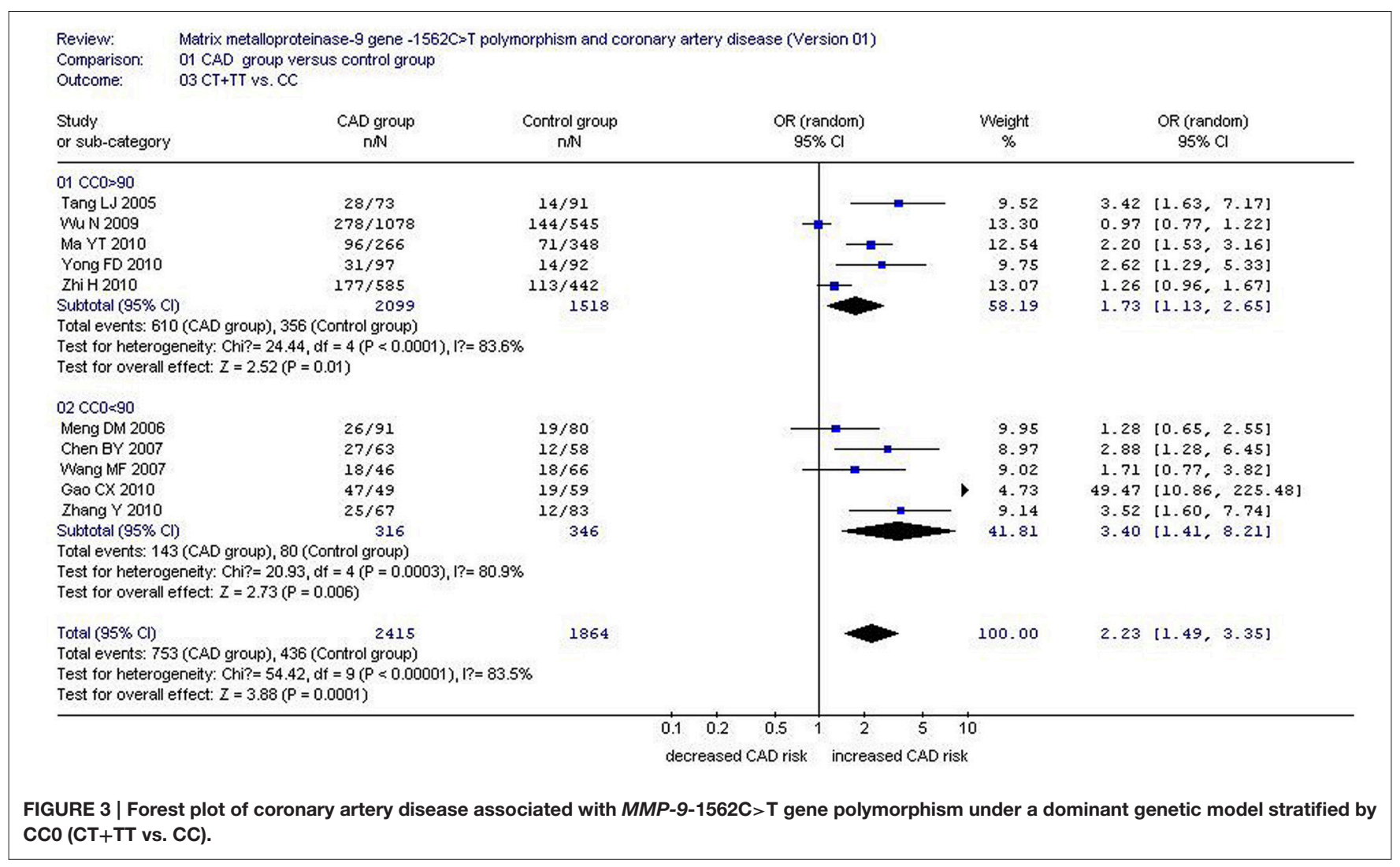

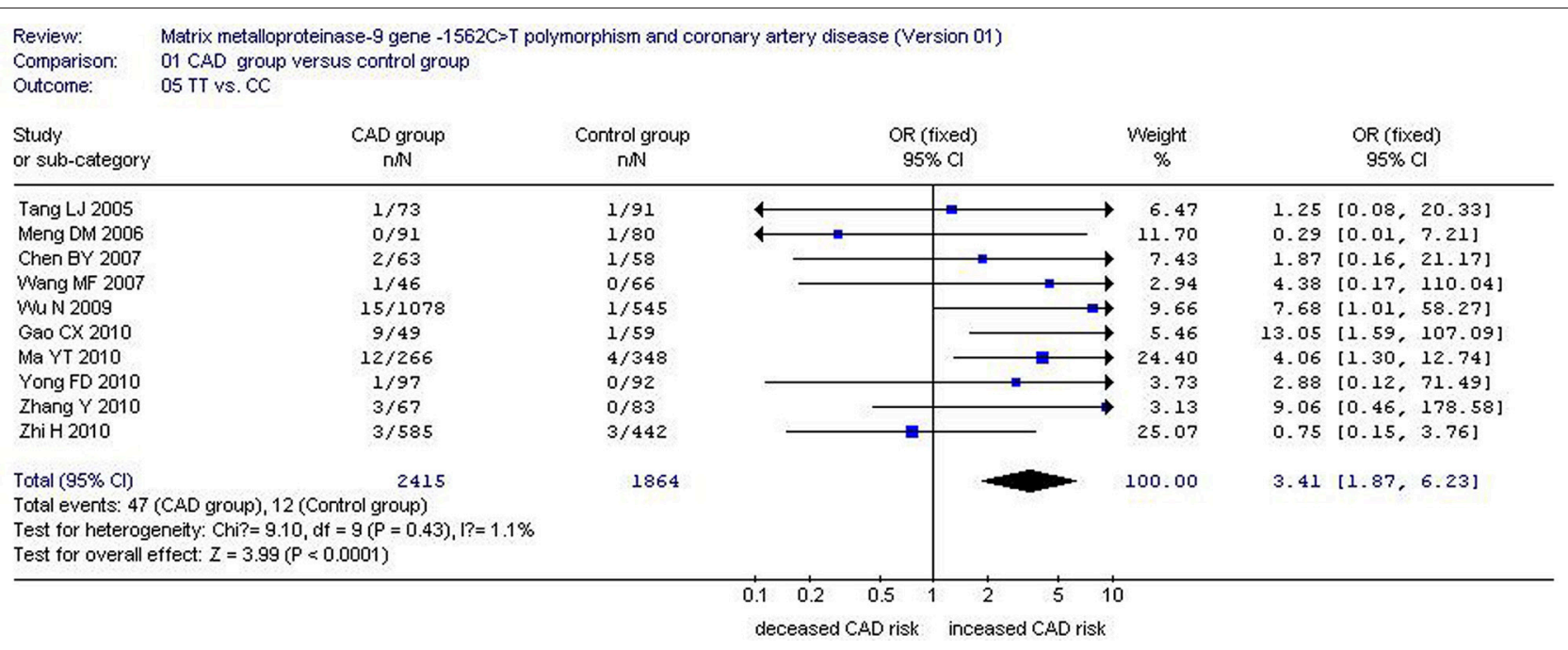

FIGURE 4 | Forest plot of coronary artery disease associated with MMP-9-1562C>T gene polymorphism under a homozygous genetic model (TT vs. CC).

heterogeneity was fond in subgroup 1 (allelic genetic model: Pheterogeneity $=0.38, I^{2}=4.0 \%$; additive genetic model: $\left.\mathrm{P}_{\text {heterogeneity }}=0.26, I^{2}=23.7 \%\right)$, but significant heterogeneity was detected in subgroup 2 (allelic genetic model: $\mathrm{P}_{\text {heterogeneity }}=$ $0.001, I^{2}=78.3 \%$; additive genetic model: $\mathrm{P}_{\text {heterogeneity }}<0.0001$, $I^{2}=85.8 \%$ ). This identifies $\mathrm{CT} 1$ as the primary confounding factor under the allelic and additive genetic models (Tables 2-4; Figures 1, 6).

Under the dominant genetic model, CC1 $(P=0.015)$, CT1 $(P=0.009)$, TT1 $(P=0.009)$, CC sample size of control group (CC0, $P=0.001$ ), CT sample size of control group (CT0, $P=$ 0.001 ), TT sample size of control group (TT0, $P=0.012$ ), and 


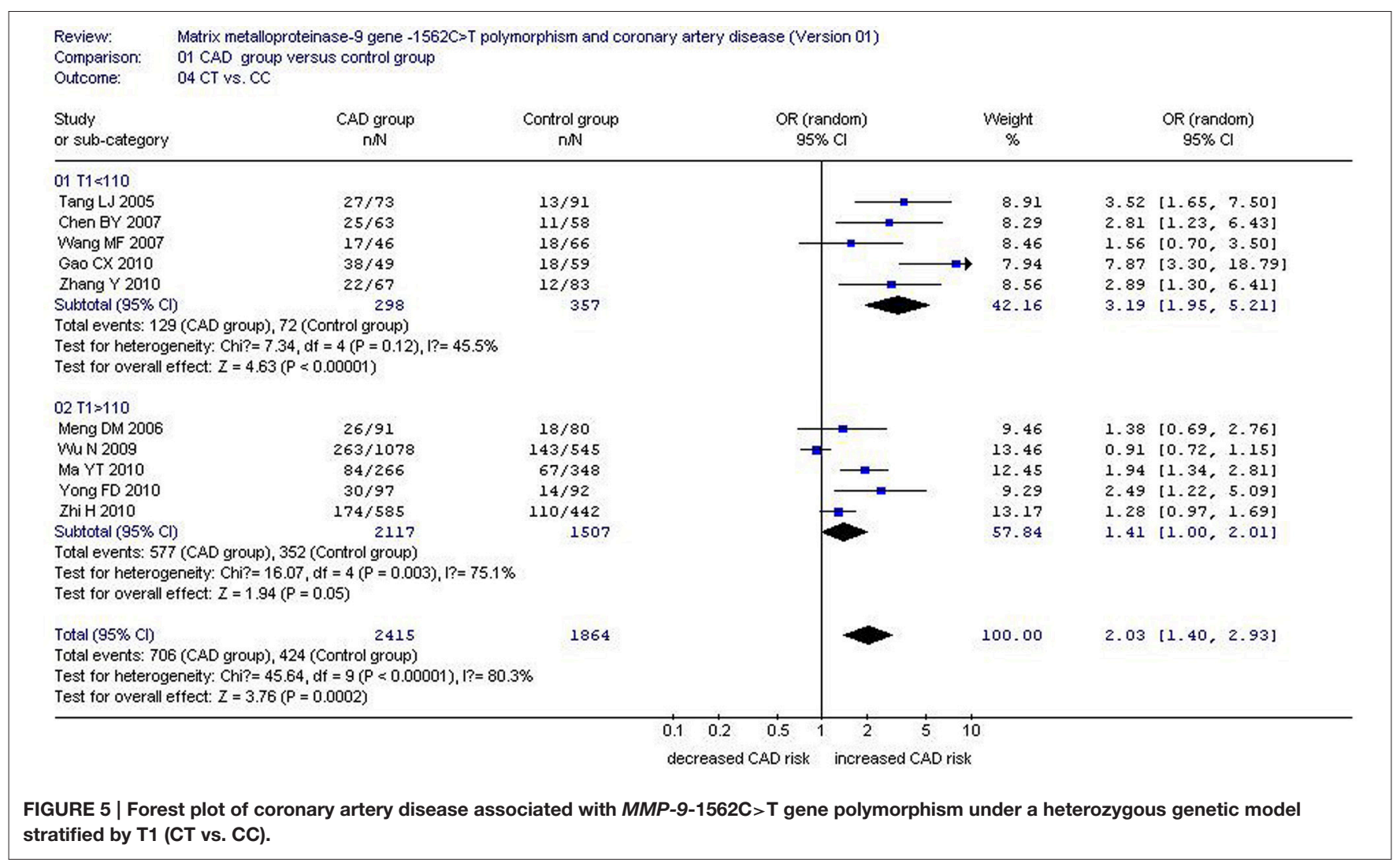

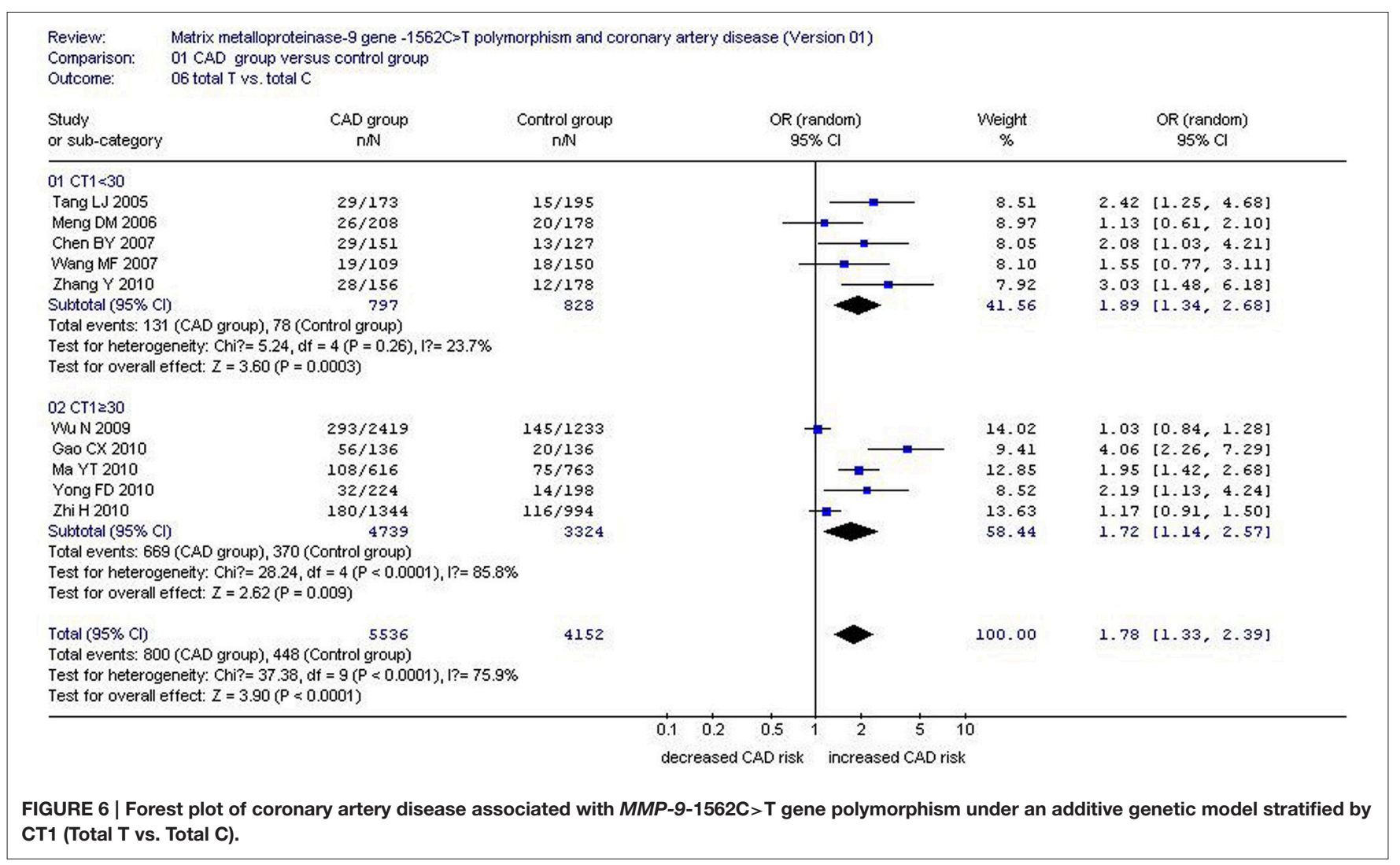


TABLE 3 | The meta-regression results among 10 studies in the Chinese population under an allelic genetic model for matrix metalloproteinase-9 gene $-1562 \mathrm{C}>\mathrm{T}$ gene polymorphism.

\begin{tabular}{|c|c|c|c|c|c|}
\hline & Coefficient & Standard Error & $T$-value & $P$-value & $95 \%$ confidence interval \\
\hline CC sample size of CAD group & -0.0081427 & 0.0011763 & -6.92 & $0.006^{\star}$ & $-0.0118862 \sim-0.0043991$ \\
\hline CT sample size of CAD group & 0.0405205 & 0.0054126 & 7.49 & $0.005^{\star}$ & $0.0232951 \sim 0.0577458$ \\
\hline TT sample size of CAD group & 0.0561608 & 0.009485 & 5.92 & $0.010^{*}$ & $0.0259754 \sim 0.0863462$ \\
\hline CC sample size of control group & 0.0423375 & 0.0098008 & 4.32 & $0.023^{*}$ & $0.0111471 \sim 0.0735279$ \\
\hline Total sample size of control group & -0.0337639 & 0.0090819 & -3.72 & $0.034^{\star}$ & $-0.0626665 \sim-0.0048613$ \\
\hline Weight(\%) & -0.2944788 & 0.0755127 & -3.90 & $0.030^{\star}$ & $-0.534794 \sim-0.0541637$ \\
\hline Cons & 2.19458 & 0.4613965 & 4.76 & $0.018^{\star}$ & $0.7262105 \sim 3.662949$ \\
\hline
\end{tabular}

${ }^{\star} P<0.05$. Coefficient: regression coefficient. The regression coefficients are the estimated increase in the InOR per unit increase in the covariates. Cons, constant item.

weight $(P=0.001)$ could partly explain the heterogeneity source. In a subgroup analysis stratified by $\mathrm{CC} 0$, significant increased CAD risk was observed in both subgroups (subgroup 1: OR: 1.73, 95\% CI: 1.13-2.65, $P=0.01$; subgroup 2: OR: 3.40, 95\% CI: $1.41-8.21, P=0.006)$. Although heterogeneity was still detected in both subgroups, it was significantly reduced in subgroup 2 $\left(\mathrm{P}_{\text {heterogeneity }}=0.0003, I^{2}=80.9 \%\right)$, suggesting that CC0 was the main confounding factor (Tables 2, 5; Figure 3 ).

Under the heterozygous genetic model, CC1 $(P=0.006)$, total sample size of CAD group (T1, $P=0.011)$, and CT0 $(P=0.004)$ could partly explain the heterogeneity. In a subgroup analysis stratified by T1, significant increase in CAD risk was detected in both subgroups (subgroup 1: OR: 3.19, 95\% CI: 1.95-5.21, $P<$ 0.00001; subgroup 2: OR: $1.41,95 \% \mathrm{CI}: 1.00-2.01, P=0.05)$. No significant heterogeneity existed in subgroup 1 any longer $\left(\mathrm{P}_{\text {heterogeneity }}=0.12, I^{2}=45.5 \%\right)$, but significant heterogeneity was still observed in subgroup $2\left(\mathrm{P}_{\text {heterogeneity }}<0.0001, I^{2}=\right.$ $85.8 \%$; Tables 2, 6; Figure 5).

\section{Sensitivity Analysis}

In the current meta-analysis, the sensitivity analysis was performed. After the $\mathrm{Wu}$ et al. was removed from the current meta-analysis, the association between MMP-9-1562C>T gene polymorphism and CAD was further strengthened under the allelic genetic model (OR: 1.72, 95\% CI: 1.35-2.19, $P=1.3 \times$ $10^{-5}, \mathrm{P}_{\text {heterogeneity }}=0.04, I^{2}=50.0 \%$; Wu et al., 2009). Removal of other studies respectively from the current studies did not change the results from the original analysis. Hence, the $\mathrm{Wu}$ et al study should be the high sensitivity study in the current meta-analysis.

\section{Bias Diagnostics}

The publication bias of the studies was evaluated by funnel plot and Egger's test. There was no visual publication bias in the funnel plot (Figure 7). No statistically significant difference was detected in the Egger's test, implying no publication bias existed in the current meta-analysis under the recessive genetic model ( $T=-0.73, P=0.487)$.

\section{DISCUSSION}

In the present meta-analysis, we found a significant association between MMP-9-1562C > T gene polymorphism and CAD in the Chinese Han population under allelic (OR: 1.60), recessive (OR: 3.05), dominant (OR: 2.23), homozygous (OR: 3.41), heterozygous (OR: 2.03), and additive genetic models (OR: 1.78). Hence, it has been concluded that in Chinese Han population, the MMP-9-1562C > T gene polymorphism may be associated with the increased CAD susceptibility among Han Chinese.

What contributed to the recent controversy over the association between MMP-9-1562C > T gene polymorphism and $\mathrm{CAD}$ ? The meta-regression used to reveal the source of the heterogeneity detected under allelic, dominant, heterozygous and additive genetic models $\left(\mathrm{P}_{\text {heterogeneity }}<0.05\right)$ suggests that patient number may have been the confounding factor. In the heterogeneity source analysis, CT1 was possibly indicated to be the main heterogeneity source under allelic and additive genetic models. Although the subgroup analysis stratified by CT1 showed a significantly increased risk of CAD in both subgroups, only one subgroup exhibited heterogeneity. Hence, CT1 was the main confounding factor contributing to the heterogeneity under the allelic and additive models. Similarly, T1 and CC0 were major confounding factors under heterozygous and dominant models, respectively. CT1, T1, CC0 may be better matched between the $\mathrm{CAD}$ and control groups under these genetic models.

Sensitivity analysis showed that our meta-analysis was most sensitive to the $\mathrm{Wu}$ et al study. Although the pooled analysis result without $\mathrm{Wu}$ et al study was different from the original result (Wu et al., 2009), they are still consistent in associating the presence of the gene polymorphism with an increased risk of CAD.

MMPs belong to a neutral protease family that contains zinc ions. MMP-9 is the leading MMP expressed and secreted by the vascular cell walls. It is also secreted by monocytes, neutrophils, vascular smooth muscle cells (VSMCs), and endothelial cells. The relative molecular weight of MMP-9 is $92 \mathrm{KD}$. In its active form its molecular weight is $84 \mathrm{KD}$. MMP-9 can degrade extensive ECM substrates, including Type IV collagen, which plays a key role in the revascularization, inflammation response, and atherosclerosis progression. Research has shown an increased MMP-9 expression level in the atherosclerotic arteries of human and animals compared to normal arteries. The MMP-9 degradation activity was most located in the shoulder regions of plaque, the lipid core margin, and micro-vessels formation regions. This suggests that MMP-9 may be associated with the coronary artery 
TABLE 4 | The meta-regression results among 10 studies in the Chinese population under an additive genetic model for matrix metalloproteinase-9 gene $-1562 \mathrm{C}>\mathrm{T}$ gene polymorphism.

\begin{tabular}{|c|c|c|c|c|c|}
\hline & Coefficient & Standard Error & $T$-value & $P$-value & $95 \%$ confidence interval \\
\hline CC sample size of CAD group & -0.0091723 & 0.0017378 & -5.28 & $0.013^{\star}$ & $-0.0147029 \sim-0.0036417$ \\
\hline CT sample size of CAD group & 0.0470359 & 0.0085234 & 5.52 & $0.012^{*}$ & $0.0199107 \sim 0.0741611$ \\
\hline TT sample size of CAD group & 0.0699275 & 0.0141987 & 4.92 & $0.016^{\star}$ & $0.0247408 \sim 0.1151142$ \\
\hline CC sample size of control group & 0.007531 & 0.0022575 & 3.34 & $0.045^{\star}$ & $0.0003466 \sim 0.0147153$ \\
\hline CT sample size of control group & -0.0376383 & 0.0137834 & -2.73 & 0.072 & $-0.0815034 \sim 0.0062267$ \\
\hline Weight & -0.4013957 & 0.139735 & -2.87 & 0.064 & $-0.8460947 \sim 0.0433033$ \\
\hline Cons & 3.40343 & 1.011299 & 3.37 & $0.044^{\star}$ & $0.1850261 \sim 6.621835$ \\
\hline
\end{tabular}

${ }^{\star} P<0.05$. Coefficient: regression coefficient. The regression coefficients are the estimated increase in the InOR per unit increase in the covariates. Cons, constant item.

TABLE 5 | The meta-regression results among 10 studies in the Chinese population under a dominant genetic model for matrix metalloproteinase-9 gene $-1562 \mathrm{C}>\mathrm{T}$ gene polymorphism.

\begin{tabular}{|c|c|c|c|c|c|}
\hline & Coefficient & Standard Error & $T$-value & $P$-value & $95 \%$ confidence interval \\
\hline Study Region & -0.0108937 & 0.0011565 & -9.42 & 0.067 & $-0.0255888 \sim 0.0038014$ \\
\hline CC sample size of CAD group & -0.0018324 & 0.0000437 & -41.90 & $0.015^{\star}$ & $-0.002388 \sim-0.0012768$ \\
\hline CT sample size of CAD group & 0.016394 & 0.0002299 & 71.31 & $0.009^{*}$ & $0.0134728 \sim 0.0193151$ \\
\hline TT sample size of CAD group & -0.0231394 & 0.0003334 & -69.40 & $0.009^{\star}$ & $-0.0273758 \sim-0.018903$ \\
\hline CC sample size of control group & 0.0214946 & 0.0000441 & 487.42 & $0.001^{\star}$ & $0.0209343 \sim 0.022055$ \\
\hline CT sample size of control group & -0.0753318 & 0.0001097 & -686.43 & $0.001^{*}$ & $-0.0767263 \sim-0.0739374$ \\
\hline TT sample size of control group & 0.0395787 & 0.0007278 & 54.38 & $0.012^{*}$ & $0.0303313 \sim 0.0488261$ \\
\hline Weight & -0.7719044 & 0.0014531 & -531.20 & $0.001^{*}$ & $-0.7903681 \sim-0.7534408$ \\
\hline Cons & 7.286745 & 0.0136083 & 535.46 & $0.001^{\star}$ & $7.113836 \sim 7.459655$ \\
\hline
\end{tabular}

${ }^{\star} P<0.05$. Coefficient: regression coefficient. The regression coefficients are the estimated increase in the InOR per unit increase in the covariates. Cons, constant item.

TABLE 6 | The meta-regression results among 10 studies in the Chinese population under a heterozygous genetic model for matrix metalloproteinase-9 gene $-1562 \mathrm{C}>\mathrm{T}$ gene polymorphism.

\begin{tabular}{|c|c|c|c|c|c|}
\hline & Coefficient & Standard Error & $T$-value & $P$-value & $95 \%$ confidence interval \\
\hline CC sample size of CAD group & -0.0710505 & 0.013625 & -5.21 & $0.006^{*}$ & $-0.1088794 \sim-0.0332215$ \\
\hline Total sample size of CAD group & 0.0614264 & 0.013634 & 4.51 & $0.011^{*}$ & $0.0235723 \sim 0.0992805$ \\
\hline CT sample size of control group & -0.0498063 & 0.0082049 & -6.07 & $0.004^{\star}$ & $-0.0725867 \sim-0.0270259$ \\
\hline Cons & 0.4150886 & 0.3419184 & 1.21 & 0.292 & $-0.534229 \sim 1.364406$ \\
\hline
\end{tabular}

${ }^{*} P<0.05$. Coefficient: regression coefficient. The regression coefficients are the estimated increase in the InOR per unit increase in the covariates. Cons, constant item.

plaque stability and myocardial infarction (Speidl et al., 2011).

The animal experiments have discovered that the atherosclerosis lesions and VSMCs intima migration in the MMP-9 gene knock-out mice were remarkably decreased than that in the wild-type mice (Ye, 2006). The clinical researches have confirmed that the high MMP-9 expression level was correlated with the premature $\mathrm{CAD}$, unstability of the coronary atherosclerosis plaque, the in-stent restenosis and arterial aneurysm formation (Jones et al., 2006). The prospective researches have shown that plasma $M M P-9$ contents can serve as the prediction indicator for the cardiovascular diseases mortality (Blankenberg et al., 2003).

MM9 is regulated primarily at the transcriptional level. The MMP-9-1562C $>\mathrm{T}$ gene polymorphism is located in crucial regulatory elements, including the 9 bp sequence GCGCAC/TGCC $\quad(-1567 \rightarrow-1559)$, a potential binding site for transcription inhibition proteins (Zhang et al., 1999). In 2002, Cho et al reported a change in bond zone structure and a weakened binding capacity between DNA and transcription inhibition protein, when the MMP-9-1562C allele was replaced by $-1562 \mathrm{~T}$ allele (Cho et al., 2002). This generates a high and low activity promoter genotype (CT/TT and CC, respectively) with increased transcription with the high activity promoter genotypes.

Increased MMP-9 expression may contribute CAD development through a number of pathways. It may promote the VSMCs proliferation and migration, promote the injured vascular remodeling, and/or promote the plaque rupture and 


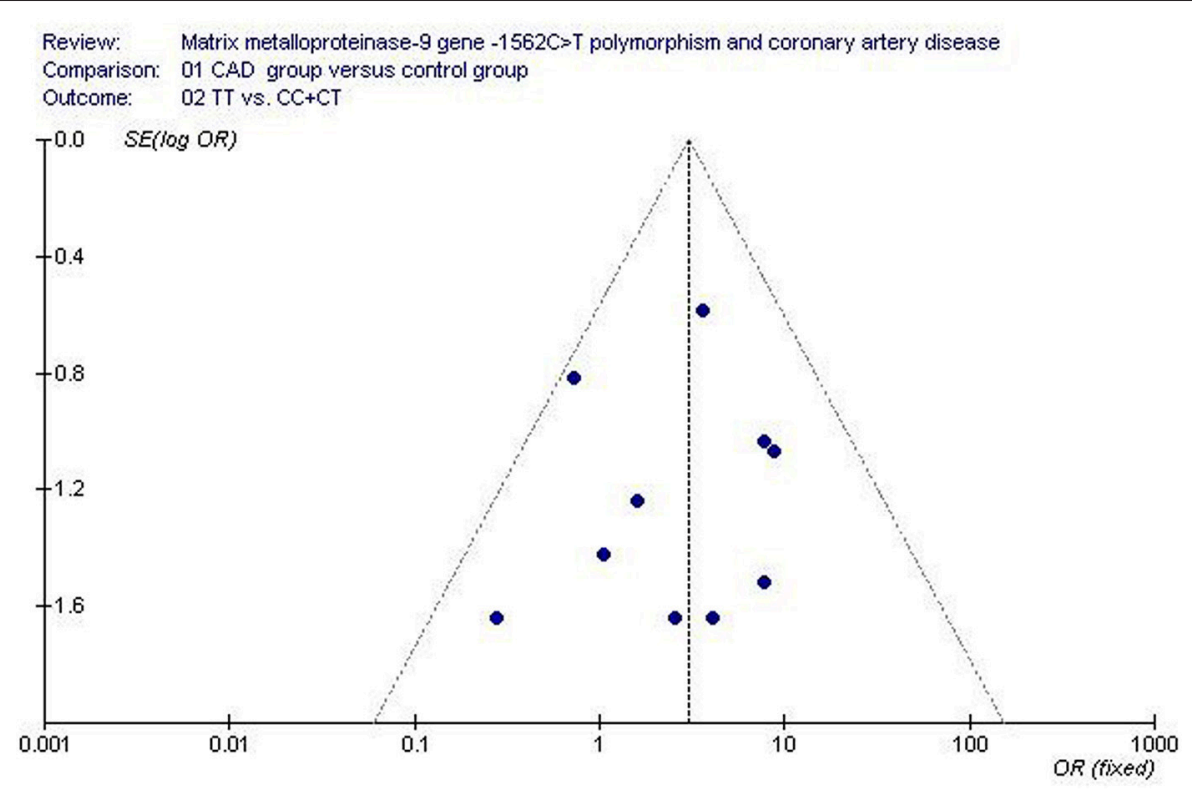

FIGURE 7 | Funnel plot for studies of the association of coronary artery disease and MMP-9-1562C>T gene polymorphism under a recessive genetic model (TT vs. CC+CT). The horizontal and vertical axis correspond to the OR and confidence limits. OR, odds ratio; SE, standard error.

lead to the thrombosis, resulting in the acute coronary syndrome known as myocardial infarction (Galis et al., 2002).

Past meta-analyses on the association of MMP-9-1562C $>\mathrm{T}$ gene polymorphism and CAD (Li et al., 2012, 2013; Niu and Qi, 2012) show weaknesses in their method. Although the distribution of genes differs between populations, these metaanalyses mixed ethnic Han Chinese with other ethnicities. The present meta-analysis, on the other hand, studies only the Han Chinese population. In addition, their initial search for manuscripts was not as comprehensive as that of the current meta-analysis, making their work less objective and credible.

However, this meta-analysis is not without limitations. The large-scale studies present in the analysis were not adequate to fully elucidate the complex relationship between the MMP-9-1562C>T gene polymorphism and CAD. The CAD susceptibility is also influenced by environmental factors, such as smoking, diabetes, dyslipidaemia, air pollution, inflammation, and psychological factors (Wang et al., 2012; Agüero et al., 2013; Parruti et al., 2013; Wichmann et al., 2013). It is quite possible that the MMP-9-1562C>T gene polymorphism interact with a risk factor that was not within the scope of this study. There are also many other MMP-9 gene polymorphisms as P574R, $\mathrm{R}+279 \mathrm{Q}$, and R668Q that influence the MMP-9 serum level (Zhi et al., 2010).

In conclusion, the current meta-analysis indicates that the MMP-9-1562T allele may increase the CAD risk among the Chinese population. This result has the potential to guide the therapy strategy for a CAD patient. Taking into account the limitations mentioned above, it remains necessary for these results to be verified by future studies.

\section{AUTHOR CONTRIBUTIONS}

YL researched data. YL and CZ wrote manuscript, researched data. YL, YZ, XY, HK, and GG reviewed/edited manuscript. YL, HG, and YQ contributed to discussion, reviewed/edited manuscript. YL, JW, and XW researched data, contributed discussion.

\section{ACKNOWLEDGMENTS}

This work was funded by the National Natural Science Foundation of China (NSFC 81100073 to YL), Excellent Young and Middle-Aged Teachers Assistance Program of Nanjing Medical University for YL, Jiangsu Overseas Research and Training Program for University Prominent Young and Middleaged Teachers and Presidents, and the Priority Academic Program Development of Jiangsu Higher Education Institutions (PAPD). Thank all our colleagues working in the Department of geriatrics, the First Affiliated Hospital of Nanjing Medical University.

\section{SUPPLEMENTARY MATERIAL}

The Supplementary Material for this article can be found online at: http://journal.frontiersin.org/article/10.3389/fphys. 2016.00212 


\section{REFERENCES}

Agüero, F., Dégano, I. R., Subirana, I., Grau, M., Zamora, A., Sala, J., et al. (2013). Impact of a partial smoke-free legislation on myocardial infarction incidence, mortality and case-fatality in a population-based registry: the REGICOR Study. PLoS ONE 8:e53722. doi: 10.1371/journal.pone.0053722

Blankenberg, S., Rupprecht, H. J., Poirier, O., Bickel, C., Smieja, M., Hafner, G., et al. (2003). Plasma concentrations and genetic variation of matrix metalloproteinase 9 and prognosis of patients with cardiovascular disease. Circulation 107, 1579-1585. doi: 10.1161/01.CIR.0000058700.41738.12

Castro, M. M., and Tanus-Santos, J. E. (2013). Inhibition of matrix metalloproteinases (MMPs) as a potential strategy to ameliorate hypertensioninduced cardiovascular alterations. Curr. Drug Targets 14, 335-343. doi: $10.2174 / 1389450111314030005$

Chen, B. Y., Li, X. Q., He, H. J., Chen, X. L., Li, Q., Kuang, X. Q., et al. (2007). Matrix metalloproteinase-9 polymorphism $(\mathrm{C} 1562 \mathrm{~T})$ and the susceptibility to unstable angina pectoris in the Han population of South China. J. Clin. Rehabilit. Tissue Eng. Res. 11, 3047-3050. doi: 10.3321/j.issn:1673-8225.2007.16.035

Cho, H. J., Chae, I. H., Park, K. W., Ju, J. R., Oh, S., Lee, M. M., et al. (2002). Functional polymorphism in the promoter region of the gelatinase $\mathrm{B}$ gene in relation to coronary artery disease and restenosis after percutaneous coronary intervention. J. Hum. Genet. 47, 88-91. doi: 10.1007/s100380200006

Cochran, W. G. (1968). The effectiveness of adjustment by subclassification in removing bias in observational studies. Biometrics 24, 295-313. doi: $10.2307 / 2528036$

DerSimonian, R., and Laird, N. (1986). Meta-analysis in clinical trials. Control. Clin. Trials 7, 177-188. doi: 10.1016/0197-2456(86)90046-2

Egger, M., Davey Smith, G., Schneider, M., and Minder, C. (1997). Bias in metaanalysis detected by a simple, graphical test. Br. Med. J. 315, 629-634. doi: 10.1136/bmj.315.7109.629

Galis, Z. S., Johnson, C., Godin, D., Magid, R., Shipley, J. M., Senior, R. M., et al. (2002). Targeted disruption of the matrix metalloproteinase-9 gene impairs smooth muscle cell migration and geometrical arterial remodeling. Circ. Res. 91, 852-859. doi: 10.1161/01.RES.0000041036.86977.14

Gao, C. X., and Wang, Y. L. (2010). Research on the matrix metalloproteinase-9 gene polymorphism and risk of coronary heart disease. Mod. Prevent. Med. 37, 328-332.

Jones, G. T., Kay, I. P., Chu, J. W., Wilkins, G. T., Phillips, L. V., McCormick, M., et al. (2006). Elevated plasma active matrix metalloproteinase-9 level is associated with coronary artery in-stent restenosis. Arterioscler Thromb. Vasc. Biol. 26, e121-e125. doi: 10.1161/01.ATV.0000226544.93089.7f

Li, J., Lu, H., Tao, F., Zhou, H., Feng, G., He, L., et al. (2013). Meta-analysis of MMP-9-1562C/T and the risk of coronary heart disease. Cardiology 124, 53-59. doi: $10.1159 / 000345772$

Li, L. M. (2007). Epidemiology. Beijing: People Medical Publishing House. 400.

Li, M., Shi, J., Fu, L., Wang, H., Zhou, B., and Wu, X. (2012). Genetic polymorphism of MMP family and coronary disease susceptibility: a metaanalysis. Gene 495, 36-41. doi: 10.1016/j.gene.2011.12.025

Ma, Y. T., Wang, L., Xie, X., Yang, Y. Y., Fu, Z. Y., Yuan. S., et al. (2010). Interactions between matrix metalloproteinase-9 polymorphism in relation to myocardial infarction in a Chinese population. Chin. J. Hypertens 18, $1167-1172$.

Mantel, N., and Haenszel, W. (1959). Statistical aspects of the analysis of data from retrospective studies of disease. J. Natl. Cancer Inst. 22, 719-748.

Meng, D. M., Mao, Y. M., Chen, Q., Geng, J., Qin, Q., Zhao, L. R., et al. (2006). Relationship between polymorphisms of matrix metalloproteinase and coronary heart disease. Tianjin Med. J. 34, 295-298. doi: 10.3969/j.issn.02539896.2006.05.003

Niu, W., and Qi, Y. (2012). Matrix metalloproteinase family gene polymorphisms and risk for coronary artery disease: systematic review and meta-analysis. Heart 98, 1483-1491. doi: 10.1136/heartjnl-2012-302085
Parruti, G., Vadini, F., Sozio, F., Mazzott, E., Ursini, T., Polill, E., et al. (2013). Psychological factors, including alexithymia, in the prediction of cardiovascular risk in HIV infected patients: results of a cohort study. PLoS ONE 8:e54555. doi: 10.1371/journal.pone.0054555

Speidl, W. S., Kastl, S. P., Hutter, R., Katsaros, K. M., Kaun, C., Bauriedel, G., et al. (2011). The complement component C5a is present in human coronary lesions in vivo and induces the expression of MMP-1 and MMP-9 in human macrophages in vitro. FASEB J. 25, 35-44. doi: 10.1096/fj.10-156083

Tang, L. J., Chen, X. F., Zhu, M., Sheng, W. F., and Jiang, J. J. (2005). Study of relations between matrix metalloproteinase-9 polymorphism (C-1562T) and acute coronary syndrome in Han population of China. Zhonghua Yi Xue Yi Chuan Xue Za Zhi 22, 313-316. doi: 10.3760/j.issn:1003-9406.2005.03.021

Tanner, R. M., Lynch, A. I., Brophy, V. H., Eckfeldt, J. H., Davis, B. R., Ford, C. E., et al. (2011). Pharmacogenetic associations of MMP-9 and MMP12 variants with cardiovascular disease in patients with hypertension. PLoS ONE 6:e23609. doi: 10.1371/journal.pone.0023609

Wang, L., Ma, Y. T., Xie, X., Yang, Y. N., Fu, Z. Y., Li, X. M., et al. (2012). Interaction between MMP-9 gene polymorphisms and smoking in relation to myocardial infarction in a Uighur population. Clin. Appl. Thromb. Hemost. 18, 72-78. doi: $10.1177 / 1076029611412365$

Wang, M. F., Xiao, C. S., Gong, S. W., Wang, R. Y., Liu, X. E., Hou, L. H., et al. (2007). Relationship study about polymorphism of matrix metalloproteinase-9 with coronary heart disease. J. Clin. Hematol. 20, 28-30. doi: 10.3969/j.issn.1004-2806.2007.01.010

Wichmann, J., Folke, F., Torp-Pedersen, C., Lippert, F., Ketzel, M., Ellermann, T., et al. (2013). Out-of-hospital cardiac arrests and outdoor air pollution exposure in Copenhagen, Denmark. PLOS ONE 8:e53684. doi: 10.1371/journal.pone.0053684

Wu, N., Lu, X., Hua, Y., Song, L., Ye, J., Li, J., et al. (2009). Haplotype analysis of the stromelysin-1 (MMP3) and gelatinase B (MMP-9) genes in relation to coronary heart disease. Ann. Hum. Genet. 73, 404-410. doi: 10.1111/j.14691809.2009.00522.x

Ye, S. (2006). Influence of matrix metalloproteinase genotype on cardiovascular disease susceptibility and outcome. Cardiovasc Res. 69, 636-645. doi: 10.1016/j.cardiores.2005.07.015

Yong, F. D., and Shi, Y. P. (2010). The Polymorphism in the MMP-9 Gene in Relation to Coronary Heart Disease. Hangzhou: Zhejiang University Press. 15.

Zhang, B., Ye, S., Herrmann, S. M., Eriksson, P., de Maat, M., Evans, A., et al. (1999). Functional polymorphism in the regulatory region of gelatinase B gene in relation to severity of coronary atherosclerosis. Circulation 99, 1788-1794. doi: 10.1161/01.CIR.99.14.1788

Zhang, Y., Wang, C. X., Dong, X., Zhu, C. Z., Duan, Z. M., Dang, Y. H., et al. (2010). Relationship between matrix metalloproteinase-2/9 polymorphism and susceptibility to premature coronary heart disease. J. Xian Jiaot. Univ. 31, $429-433$.

Zhi, H., Wang, H., Ren, L., Shi, Z., Peng, H., Cui, L., et al. (2010). Functional polymorphisms of matrix metallopeptidase- 9 and risk of coronary artery disease in a Chinese population. Mol. Biol. Rep. 37, 13-20. doi: 10.1007/s11033009-9482-x

Conflict of Interest Statement: The authors declare that the research was conducted in the absence of any commercial or financial relationships that could be construed as a potential conflict of interest.

Copyright $\odot 2016$ Li, Yang, Zhou, Gong, Geng, Kim, Zhou, Qian, Wang and Wu. This is an open-access article distributed under the terms of the Creative Commons Attribution License (CC BY). The use, distribution or reproduction in other forums is permitted, provided the original author(s) or licensor are credited and that the original publication in this journal is cited, in accordance with accepted academic practice. No use, distribution or reproduction is permitted which does not comply with these terms. 